\title{
Análisis de las capacidades de empresas de servicios públicos en el Área Metropolitana de Bucaramanga en su proyección a distrito
}

\section{Analysis of the capacities of public service companies in the Metropolitan Area of Bucaramanga in its projection to the district}

DOI: $10.46932 / \mathrm{sfjdv} 2 \mathrm{n} 4-070$

Received in: March 1st, 2021

Accepted in: May 30th, 2021

\author{
Fernando Polo Acevedo \\ Magister en Administración Pública Territorial \\ Escuela Superior de Administración Publica \\ Bucaramanga, Colombia \\ E-mail: polo.fernando@hotmail.com \\ Francisco Yesid Triana Castillo \\ Magister en Administración Pública Territorial \\ Escuela Superior de Administración Publica \\ Bucaramanga, Colombia \\ E-mail: Yesid.triana@hotmail.com \\ Carmen Elisa Therán Barajas \\ Magister en Administración \\ Escuela Superior de Administración Publica \\ Bucaramanga, Colombia \\ E-mail: Ctheran59@yahoo.es

\section{Narcy Carolina Prieto Cuentas} \\ Magister en administración de empresas \\ Programa de Logística y Mercado Organizacional \\ Fundación Universitaria Comfenalco Santander \\ Grupo de investigación GIPS \\ E-mail: Carolinaprieto86@hotmail.com
}

\section{RESUMEN}

La presente investigación, producto de un trabajo de maestría de la Escuela superior de administración pública, en sinergia con la Fundación Universitaria Comfenalco Santander, establece en primer lugar una caracterización de las principales empresas de servicios públicos de acueducto, aseo y alcantarillado del Área Metropolitana de Bucaramanga (AMB), desde sus capacidades de prestación de servicios en la actualidad. La segunda parte muestra el comportamiento económico de la empresa de servicio público con proyección triple $\mathrm{A}$ del $\mathrm{AMB}$, en un posible escenario a futuro de conformación de distrito metropolitano. La tercera parte, desarrolla la prospectiva de la empresa de servicio público, con la capacidad de convertirse en triple A, suministrando servicios continuos con cobertura del AMB, en sinergia con otras entidades, permitiendo hacer frente al crecimiento poblacional, el desarrollo territorial y la proyección a distrito metropolitano, definiendo las variables estratégicas sobre las que se deben actuar en el largo plazo, las cuales tienen una alta influencia en el sistema y los posibles escenarios a futuro de la organización, de forma que sea una guía para el proceso de planeación y toma de decisiones, que incluya los posibles cambios en el entorno producto de la dinámica regional. 
Finalmente, el estudio se podría convertir en un insumo para la propuesta de la Cámara de Comercio de Bucaramanga, sobre la viabilidad de establecer una alternativa de cohesión en el área de servicios públicos para los municipios que conforman el Área Metropolitana de Bucaramanga en su proyección a distrito.

Palabras Clave: Empresas de servicios públicos, Proyección, distrito metropolitano, alianza estratégica.

\begin{abstract}
This research first establishes a characterization of the main aqueduct, cleaning, and sewerage public service companies of the Bucaramanga Metropolitan Area (AMB), from their current service provision capabilities. The second part shows the economic behavior of the public service company with triple A projection of the $\mathrm{AMB}$, in the territorial development and the projection to the metropolitan district. The third part develops the prospective of the triple A public service companies of the AMB, in front of the territorial development and the projection to the metropolitan district, defining the strategic variables on which they must act in the long term, which have a high influence in the system and the possible future scenarios of the organization so that it is a guide for the planning and decision-making process, which includes possible changes in the environment as a result of territorial development.

Finally, the study could become an input for the Bucaramanga Chamber of Commerce proposal on the feasibility of establishing a cohesion alternative in the public services area for the municipalities that make up the Bucaramanga Metropolitan Area.
\end{abstract}

Keywords: Public service companies, Projection, metropolitan district, strategic alliance

\title{
1 INTRODUCCIÓN
}

La ciudad de Bucaramanga, hoy en día, es reconocida como el mayor centro de negocios del nororiente colombiano. Posee un área metropolitana, que ocupa el 5\% del territorio del departamento, donde se concentra el $50.51 \%$ de la población, conformada por los municipios de Floridablanca, Girón y Piedecuesta, ubicados en la provincia de Soto. Donde el $96 \%$ de su población es urbana y se concentra la industria manufacturera del departamento de Santander, especializándose en el sector terciario, principalmente, en servicios de educación, salud, comercio, hoteleros y financieros (Universidad Industrial de Santander, 2018). Ocupando el cuarto puesto en el escalafón de competitividad del país (Ramírez \& Aguas , 2015), se encuentra entre las 10 ciudades que reclama especial atención, debido a su fuerte dinámica de crecimiento económico y su capacidad de gestión institucional y administrativa, por lo tanto, debe ser estudiada y apoyada dentro de este contexto, desde la academia y las organizaciones del sector público y privado.

Por lo anteriormente mencionado existe un interés en la región de avanzar hacia la conformación de un distrito metropolitano y para ello se requieren procesos de integración o de apoyo entre las diferentes empresas que prestan servicios en cada municipio y de ahí la importancia de analizar estas empresas para establecer aspectos que permitan trabajar coordinadamente entre ellas, con el objetivo de fortalécelas desde una visión de consolidación de un distrito metropolitano. Donde "El apoyo técnico y financiero a 
estas ciudades debería plantear como requisito el trabajo conjunto entre las ciudades de una aglomeración, es decir, entre las ciudades de mayor interrelación e interdependencia” (Banco Interamericano de Desarrollo, 2015, p. 19), razón por la cual se requieren investigaciones que apunten al análisis de las capacidades técnicas y financieras de las empresas de servicios en el área metropolitana, incluyendo la proyección a futuro de las mismas como respuesta al desarrollo poblacional y territorial en la consolidación de un distrito, que permita un trabajo sinérgico entre municipios, propendiendo por la mejora en la logística de aprovisionamiento de servicios y por ende el bienestar de sus habitantes.

En el área metropolitana, la oferta de servicios públicos domiciliarios relacionados con acueducto, alcantarillado y aseo, está a cargo de siete empresas prestadoras del servicio de aseo, tres de acueducto y tres de alcantarillado (Comisión Regional de Competitividad Santander Competitivo, 2013). Donde se puede observar, que no hay una articulación conjunta de las entidades territoriales para la prestación de este tipo de servicios públicos.

Una de las necesidades en Bucaramanga y su AMB, es la cohesión de las empresas de servicios públicos, las cuales operan de forma independiente, donde se evidencia la necesidad de implementar instrumentos de planificación urbana, para pasar a la conformación de un Distrito Metropolitano, desde una visión holística del AMB, como precedente, para la conformación de un Distrito Metropolitano, permitiendo una mejora en la gestión del territorio, dándole la oportunidad de acceso a una mayor inversión por parte de la nación y beneficios adicionales. Sin embargo, en los ámbitos territoriales ha sido una tarea difícil gestionar e implementar proyectos que se enfoquen en la correspondiente articulación entre municipios, que le permitan al área metropolitana la obtención de una resolución de distrito metropolitano. Donde una de las necesidades para que esto sea posible es la cohesión de las empresas de servicios públicos, las cuales en la actualidad funcionan de manera independiente en su mayoría, generando una gestión menos eficiente en la prestación de los servicios públicos. (Comisión Regional de Competitividad Santander Competitivo, 2013).

Todo lo anterior requiere como punto de partida para la planeación e implementación de estrategias de cohesión, en empresas del sector público el análisis de las capacidades técnicas y financieras de las organizaciones de prestación de servicios públicos, dentro de un entorno cambiante y en desarrollo, que se proyecta a distrito metropolitano. Requiriendo para la implementación de estos desarrollos el liderazgo de las ciudades y gobiernos de cada municipio. (Suárez, 2021)

\section{METODOLOGÍA}

La presente investigación desde el objeto de estudio es de tipo descriptivo, porque se realizó para conocer las capacidades técnicas y tecnológicas de las empresas de servicios públicos de acueducto aseo 
y alcantarillado más importantes en el AMB de forma que permitió establecimiento de una empresa con proyección a triple A, que suministre los servicios de forma continua a los municipios de Bucaramanga, Piedecuesta, Floridablanca y Girón, dentro del contexto de una posible consolidación de distrito metropolitano a 2025 y su proyección a futuro, desde el establecimiento de los diferentes escenarios en que esta puede incurrir a 2025.

Para tal fin, en una fase preliminar, se realizó una caracterización de las empresas más relevantes de Bucaramanga y AMB que prestan los servicios de aseo, acueducto y alcantarillado, con la ayuda de un grupo de expertos para la definición de una empresa con proyección a triple A que preste un servicio continuo en el AMB. En una primera fase se evaluó la capacidad técnica y económica de la empresa con proyección triple A, frente a la evolución del área metropolitana de Bucaramanga, estableciendo que la empresa Acueducto Metropolitano de Bucaramanga, es la organización que cuenta con la capacidad para proyectarse a triple A. En una segunda fase se realizó el análisis del comportamiento económico y financiero de la empresa Acueducto Metropolitano de Bucaramanga (amb), para determinar si la empresa cuenta con la solidez financiera que requiere una proyección a triple A. En una tercera fase se realiza la proyección de los escenarios a futuro de dicha empresa teniendo en cuenta los tres campos de la prospectiva: La planeación estratégica, el análisis de las políticas y él estudio futuro.

El estudio cuenta con información primaria a partir de entrevistas a profundidad y bases de datos de las empresas prestadoras de servicios públicos triple A del Área Metropolitana de Bucaramanga, además de fuentes del DANE y la superintendencia de Servicios públicos y la Contaduría General de la Nación.

La información secundaria se tomó información apoyada en estudios y propuestas realizadas por universidades y organismos multilaterales, referenciados en la investigación, a partir de la cual se definieron los lineamientos a seguir para el presente estudio.

Para el desarrollo de la caracterización de las empresas de servicio público triple A del Área Metropolitana de Bucaramanga, desde sus capacidades de prestación de servicio actual, se tuvo en cuenta la organización administrativa y estructura de las empresa más representativas de servicios municipal del Área Metropolitana de Bucaramanga, las cuales son: El Acueducto Metropolitano De Bucaramanga amb E.S.P, la Piedecuestana de Servicios Públicos, E.S.P, la Empresa Municipal de Aseo, Acueducto y Alcantarillado de Floridablanca - EMAF E.S.P, la Empresa Pública de Alcantarillado de Santander EMPAS S.A E.S.P la empresa de aseo de Bucaramanga EMAB E.S.P, y la Empresa de Servicios Públicos de Santander ESANT S.A. ESP. Donde se realizó la caracterización de las empresas de forma que se establece que la organización que cuenta la capacidad para proyectarse a triple A es el amb. 
Para el análisis del comportamiento económico de la empresa de servicio público con proyección triple A del Área Metropolitana de Bucaramanga, frente al desarrollo territorial y la proyección a distrito metropolitano, se revisaron los informes anuales entregados a la superintendencia de servicios públicos y los presupuestos ejecutados y presentados ante la contaduría nacional, y a partir de los estados financieros de la organización.

En cuanto a la determinación del potencial de desarrollo de las empresas de servicio público con proyección triple A del Área Metropolitana de Bucaramanga, frente al desarrollo territorial y la posibilidad de conformación de un distrito metropolitano, se toman los desarrollos anteriores para empresas triples A y la capacidad para proyectarse en la integración de los tres servicios; y se contrastan frente a teorías del desarrollo territorial. Para esto se realizó una proyección a partir de la herramienta prospectiva MIC MAC, estableciendo los posibles escenarios a futuro que tiene la organización, teniendo en cuenta la posibilidad de consolidación a distrito.

\section{DESARRIOLLO Y DISCUSIÓN.}

3.1 SELECCIÓN DE LA EMPRESA QUE POSEE LAS MEJORES CONDICIONES PARA LA CONSOLIDACIÓN DE UNA ORGANIZACIÓN TRIPLE A EN UNA PROYECCIÓN A DISTRITO METROPOLITANO.

\subsubsection{Análisis organizacional:}

La empresa Piedecuestana de servicios públicos, única prestadora de servicios públicos domiciliarios de carácter público triple A en la región nororiente; posee una estructura organizacional lineo funcional, con centros de poder establecidos, que se relación entre sí para el cumplimiento de los objetivos estratégicos; donde la máxima autoridad es la junta directiva, seguida de la gerencia general.

Se observa una departamentalización por funciones, cuya finalidad es la de agrupar las actividades similares y las personas que las ejecutan en unidades específicas de trabajo con una misma ubicación. La empresa cuenta con cuatro departamentos: Administrativo y financiero, planeación e infraestructura, técnica y de operaciones y comercial, y no se establece una línea de staff desde su organigrama.

El sistema de acueducto es el principal motor de la empresa Piedecuestana de Servicios Públicos, con la cobertura del $100 \%$ de la zona urbana del Municipio y con la expansión en algunos sectores considerados como semiurbanos en cercanías al casco principal de Piedecuesta.

Actualmente la empresa cuenta con una Planta de Tratamiento de Agua Potable, denominada "La Colina", la cual capta 450 lps (litros por segundo) del Rió de Oro y desde donde se distribuye por medio de un sistema de presión por gravedad al Municipio de Piedecuesta. 
Cuenta con un crecimiento en la cobertura del servicio de Acueducto del $3 \%$ anual y un comportamiento que representa fuerza y posicionamiento para la empresa, con un crecimiento del $35 \%$ de usuarios entre 2015-2019, para un total de 119.642 usuarios y 104.580 subsidios y las tarifas de agua potable más económicas de la región. Empresa que cuenta con dos reconocimientos a nivel regional y nacional en la categoría de mejor gestión y sostenibilidad del medio ambiente.

Actualmente la empresa Piedecuestana de Servicios Públicos ESP recibe de la Administración Municipal los recursos necesarios para subsidiar una parte del servicio a estratos 1 con $70 \%, 2$ con el $40 \%$ y 3 del $15 \%$.

El servicio de Alcantarillado que presta la empresa Piedecuestana de Servicios Púbicos ESP es el encargado de proporcionar la conducción de las aguas servidas (sanitarias) y pluviales (lluvias) en el Municipio de Piedecuesta al 100\% del sector urbano.

Para la prestación de este servicio de aseo la empresa Piedecuestana de Servicios Públicos ESP cuenta con cinco carros compactadores propios, cuyas cajas compactadoras son modelo 2013- 2014, lo que permite garantizar calidad y eficiencia a la hora de la recolección de los desechos.

Es importante resaltar que la organización cuenta con una infraestructura, en su mayoría, propia, para la prestación de sus servicios de forma eficiente. Adicional a esto la empresa se proyecta para un desarrollo organizacional, enfocado no solo en el crecimiento poblacional, sino también, desde la inversión en la infraestructura requerida para la minimización de la carga contaminante generada en el Municipio. (Piedecuestana de servicios públicos e.s.p, 2019).

Por lo anteriormente mencionado la organización brinda un servicio triple A en el municipio de Piedecuesta, de forma eficiente y con gran cobertura, la cual se puede consolidar como un aliado estratégico en la consolidación de una empresa triple A en el largo plazo, proyectando este modelo para la prestación de servicios en los municipios de Bucaramanga, Girón y Floridablanca. Para la consolidación de una única empresa triple A prestadora de servicios en una proyección a distrito metropolitano.

El Acueducto metropolitano de Bucaramanga S.A. (Amb), es una organización de tipo lineo funcional, donde la responsabilidad se transmite a partir de un jefe para cada área, y la mayor autoridad la ejerce la asamblea, seguida de la junta directiva, el departamento de control de gestión y el gerente general. Al igual que la Piedecuestana de Servicios, establece una departamentalización por funciones. Es importante resaltar que, a diferencia de la empresa anterior, la institución cuenta con cinco líneas de staff: El equipo de servicios empresariales, equipos de servicios financieros, equipo de atención al cliente, equipo de servicios técnicos y el equipo de gestión de ingresos.

Adicional a esto la empresa cuenta con una línea de temporalidad para funciones en el área de producción, única en las empresas estudiadas. Esta estrategia le permite contar con la cantidad de personal 
adecuado según los requerimientos del amb, de forma que se aumenta la eficiencia, permitiendo la adaptación al entorno a partir de su talento humano y la flexibilidad de su organigrama.

Es importante destacar que la organización presenta un crecimiento acelerado en los últimos años, ampliando su cobertura y su capacidad de prestación del servicio de acueducto en los municipios de Bucaramanga, Floridablanca y Girón, garantizando un suministro de agua constante y un aumento del nivel de satisfacción de sus usuarios de más del $80 \%$ a partir de su infraestructura en crecimiento, fortalecida con inversiones en equipos de captación, plantas de tratamiento, tanques de almacenamiento y redes de distribución; y el desarrollo de su talento humano (Amb, 2014) Adicional a esto desde su dirección estratégica la institución se proyecta en la adquisición de nuevas unidades estratégicas de negocios.

Esta empresa posee la estructura organizacional, la infraestructura, el desarrollo del talento humano y sobre todo un direccionamiento estratégico que apuesta por la ampliación de sus servicios y el establecimiento de alianzas estratégicas desde su planeación. Por todo lo anterior se debe realizar el análisis del comportamiento económico y financiera de esta empresa y la valoración de sus escenarios a futuro teniendo en cuenta la conformación de un distrito metropolitano y su proyección a triple A.

La Empresa Municipal de Aseo, Alcantarillado y Acueducto de Floridablanca (EMAF), cuenta con una estructura jerárquica definida de tipo lineo funcional, donde la máxima autoridad la ejerce la junta directiva y no los accionistas, al igual que la Piedecuestana, de igual forma, no se observa una línea de staff, definida desde el organigrama. Esta presenta una departamentalización por funciones, las cuales se dividen en un área de proyectos, un área de funciones y un área operativa.

Esta empresa cuenta con un patrimonio de $\$ 76.908$ a 2013 y su infraestructura solo le permite prestar el servicio de aseo en el municipio de Floridablanca (Alcaldía municipal de Floridablanca, 2014), razón por la cual no se tiene en cuenta para su proyección a triple A. Esta organización requiere en primera instancia de un direccionamiento estratégico que apunte a la consolidación y mejoras en el servicio prestado en términos de cobertura, capacidad empresarial y servicio al cliente.

La Empresa de Aseo de Bucaramanga S.A. EMAB, es una organización de tipo lineo funcional. donde la mayor autoridad pertenece a la asamblea de accionistas, seguido por la junta directiva y el gerente. Presenta un organigrama departamentalizado por funciones con dos líneas de staff, estratégicamente desarrolladas para la revisoría fiscal y el auditor externo.

Esta presta los servicios de aseo al municipio de Bucaramanga, en estos momentos la institución concentra sus esfuerzos en el aprovechamiento de los residuos sólidos y su meta se centra en pasar de un reciclaje formal del 2\% al 30\% para el año 2023. Para esto instaló la Estación de Clasificación y Aprovechamiento de residuos sólidos (ECA), una planta de separación de productos reciclables 
semiautomatizada y adquirió nuevos camiones. (Alcaldía de Bucaramanga, 2019). Adicional a esto es una de las empresas más importantes para la ciudad, en el liderazgo de los procesos estratégicos para el cierre de El Carrasco y el reemplazo del sitio de disposición final de residuos sólidos, estableciendo un nuevo relleno sanitario regional.(Alcaldia de Bucaramanga, 2019), para evitar las multas del 2017 de 530 millones de pesos, por fallas en la disposición final de los residuos en el relleno sanitario y la realización de una cobertura diaria de los residuos, y el incorrecto manejo de lixiviados y control de vectores y roedores. (Blu Radio Bucaramanga, 2018)

Por lo anterior se establece que la organización no presenta interés en una proyección triple A, sino que debe centrar su interés en el desarrollo de proyectos prioritarios como lo son el reciclaje y la disposición final de los residuos sólidos del municipio, de forma que logre un impacto en la región desde procesos que aporten al desarrollo sostenible y de esta forma lograr su reconocimiento y crecimiento.

La Empresa Pública de Alcantarillado de Santander EMPAS S.A E.S.P., es de tipo lineo funcional donde la mayor autoridad recae sobre la asamblea general de accionistas, seguido de la junta directiva y la gerencia general, las cuales están bajo la regulación del revisor fiscal que se establece a partir de una línea de staff.

Es importante resaltar que esta empresa opera de forma eficiente y en comodato las redes de alcantarillado en los municipios de Bucaramanga, Floridablanca, Girón y casi la totalidad del departamento. A su vez esta empresa tiene características para su proyección a triple A, sin embargo esta organización establece un único servicio especializado como operador, para todo el departamento y se proyecta para la ampliación de la cobertura del servicio prestado y su capacidad, y no para la prestación de servicios complementarios desde áreas específicas del región, por esto se establece que esta institución es clave para una alianza estratégica, con otra institución complementaria a su servicio que tenga la proyección a triple A. Desde su estructura organizacional EMPAS posee una departamentalización por funciones, organizada en seis departamentos, secretaria general, subgerencia comercial y tarifaria, subgerencia administrativa y financiera, subgerencia alcantarillado subgerencia de planeación e informática y subgerencia integral de aguas y residuos. (EMPAS, 2019)

La empresa de servicios públicos de Santander ESANT S.A. ESP, es una organización de tipo lineo funcional, cuya máxima autoridad la ejerce la asamblea corporativa, la junta directiva y la revisoría fiscal, seguido del comité directivo y la gerencia general respectivamente. Adicional a esto la empresa se encuentra departamentalizada por funciones distinguiéndose la dirección administrativa y financiera, la dirección de proyectos, la dirección de operaciones, la dirección jurídica y por último la dirección de planeación. (ESANT, 2018). 
En este orden de ideas se establece que todas las empresas estudiadas proseen una estructura organizacional claramente definida de tipo lineo funcional, departamentalizada por funciones con sus respectivos centros de poder. Adicional a esto las empresas EMAF y Piedecuestana de Servicios son las únicas empresas que no presentan como máxima autoridad la asamblea de accionistas.

\section{Infraestructura:}

Es importante resaltar que la infraestructura más sólida en cuanto a una proyección a triple A la posee, en primer lugar la empresa Piedecuestana de Servíos Públicos, que actualmente suministra los servicios de Aseo, Acueducto y Alcantarillado a los habitantes de Piedecuesta y el Amb que suministra el servicio de acueducto al municipio de Bucaramanga, seguida de la empresa Empas que opera las redes de alcantarillado, respectivamente en los municipios complementarios del Área Metropolitana, Bucaramanga, Floridablanca y Girón.

Proyecciones empresariales: Desde su planeación estratégica la Piedecuestana de Servicios Públicos, no planea la adquisición de nueva unidad estratégica de negocio, pero si proyecta un liderazgo a nivel regional en sus servicios prestados, debido a que la organización presta un servicio integral a todo el municipio de Piedecuesta en cuanto a aseo, acueducto y alcantarillado.

En cuanto al Amb, se observa que tienen como propósitos empresariales, el desarrollo de actividades complementarias que integran el área Metropolitana de Bucaramanga, adicional a esto la organización con sus filiales ampliará la prestación de los servicios relacionados con la gestión integral del agua. La institución establece como prioridad la rentabilidad, y el bienestar social, desde las operaciones de nuevos negocios y la Asociación y conformación de consorcios, uniones temporales o cualquier tipo de asociación que la ley permita. Para esto la empresa cuenta con una sólida política de capacitación del personal y una estructura flexible que permite permear estos cambios para adaptarse al entorno.

De acuerdo a la caracterización realizada en el desarrollo del primer objetivo se establece que, la Empresa Municipal De Aseo, Alcantarillado y Acueducto De Floridablanca EMAF, la Empresa Pública de Alcantarillado de Santander EMPAS S.A E.S.P, la Empresa de Aseo de Bucaramanga S.A EMAB, y la empresa de servicios públicos de Santander ESANT S.A. ESP, no cuentan con la infraestructura propia requerida para una proyección triple A en el momento y desde su planeación estratégica no existen objetivos ni políticas claras de adquisición de nuevas unidades estratégicas de negocios.

Por lo anteriormente analizado, se continuará el desarrollo de la investigación con la empresa Acueducto Metropolitano de Bucaramanga, la cual posee una estructura organizacional sólida, con unas 
políticas claras en cuanto a capacitación del talento humano, alta eficiencia organizacional y una proyección de crecimiento a nivel regional y a la adquisición de unidades estratégicas y formación de asociaciones.

\subsection{ANÁLISIS DEL COMPORTAMIENTO ECONÓMICO DE LA EMPRESA DE SERVICIO PÚBLICO CON PROYECCIÓN TRIPLE A DEL ÁREA METROPOLITANA DE BUCARAMANGA, FRENTE AL DESARROLLO TERRITORIAL Y LA PROYECCIÓN A DISTRITO METROPOLITANO.}

\subsubsection{Comportamiento económico de la empresa de acueducto metropolitano de Bucaramanga amb}

Para el cierre de la vigencia 2018, el Amb S.A. ESP presenta indicadores favorables como resultado de la aplicación de políticas trazadas desde la Junta Directiva y la Gerencia General, tales como, el margen EBITDA el cual alcanza un valor de 47,64\%, ingresos operacionales totales para el año de $\$ 157.476,5$ millones y una utilidad operacional de $\$ 41.806,8$ millones, cerrando con una utilidad neta después de impuestos de \$34.181millones.

El pasivo total presenta una disminución del 7,14\%, equivalente a $\$ 27.733$ frente a la vigencia anterior, debido principalmente a las amortizaciones de los créditos de largo plazo con el sistema financiero. El Patrimonio, al cierre del ejercicio, registra un valor de $\$ 576.258,3$ millones con un incremento del 6,03\%, equivalente a un nivel de propiedad de los Accionistas sobre el Activo de 61,50\%.

Con el fin de asegurar la continuidad y cobertura en el Área de Prestación de Servicio, se dio cumplimiento al Plan de Obras e Inversiones reguladas (POIR), en lo relativo a programas de reposición y ampliación de la capacidad de las redes de acueducto, el control de las pérdidas técnicas, la optimización de presiones en los distritos hidráulicos, automatización en los sistemas de filtración de las plantas de tratamiento y la actualización tecnológica operacional. En la vigencia se registró una producción superior a los 70 millones de m3 entregados a la red de distribución, de los cuales el 41,4\% es el aporte del sistema Tona, el 23,9\% del sistema Suratá y el 19,3\% del sistema Frío. (amb, 2018, p.3).

Los Estados Financieros del amb S.A. ESP registran buenos resultados producto de la gestión integral del servicio público domiciliario de acueducto, reportando ingresos operacionales totales para el año de \$157.476 millones y una utilidad operacional de \$41.807 millones, y una utilidad neta después de impuestos de $\$ 34.181$ millones.

Adicional a esto "El Activo Total al cierre de 2018 se incrementó en \$5.076 millones, 0,54\% más que en 2017, consolidando el Total de Activos del amb S.A. ESP en $\$ 936.862$ millones. Resaltando que los Pasivos registran un detraimiento del 7,14\% equivalente a $\$ 27.733$ millones, para un pasivo total de $\$ 360.603$ millones. El Patrimonio al cierre del ejercicio registra un valor de \$576.258 millones con un 
incremento del 6,04\%; teniendo encuentra estos resultados, el nivel de propiedad de los Accionistas sobre el Activo es de 61,51\%”. (amb, 2018, p.7)

El estado de resultado integra, cierra con una Utilidad Neta a 31 de diciembre de 2018 de $\$ 34.181$ millones, lo que representa un crecimiento del 18,33\% frente al mismo periodo del 2017, resultado que se explica fundamentalmente en un buen comportamiento en la eficiencia operacional y el menor valor de provisión realizada en este ejercicio para la provisión de impuesto de renta y sobretasa. Es importante resaltar que la organización está realizando una buena utilización de sus recursos, provenientes en más de un $92 \%$ de las tarifas cobradas por consumo y cargo fijo de sus usuarios, dentro de la normativa legal vigente, impuesta por la CRA. En la empresa se evidencia un crecimiento en la utilidad neta obtenida al final de cada periodo contable desde 2014 y un patrimonio en crecimiento en los últimos tres años analizados (amb, 2018). Todo lo anterior demuestra un potencial de crecimiento para la institución y la capacidad para adquirir nuevas unidades estratégicas de negocios desde su sólido desarrollo financiero y su planeación estratégica.

“En adición a esto el $92,15 \%$ de los ingresos totales del amb corresponde al cargo por consumo y cargo fijo que presento un crecimiento del 4,24\% respecto de la vigencia anterior”. (amb, 2018, p.19) y "los costos por actividad del servicio de acueducto representan el 97\% de los costos de la operación y se distribuyen así: Abastecimiento $\$ 30.672$ millones con un porcentaje de participación del 38,44\% y un incremento del 16,36\% frente a la vigencia anterior, distribución por valor de \$28.171 millones que corresponde a 35,30\% y un incremento del 3.37\%". (amb, 2018, p.20).

Por otro lado los rubros de mayor representación se concentran en "salarios y prestaciones sociales, con un porcentaje de participación del 32,35\% frente al valor total de este concepto, con una variación porcentual del 5,55\% respecto al año inmediatamente anterior, resultado inferior al incremento salarial pactado por convención del 5,9\%, el rubro de depreciaciones y amortizaciones representa el 27,77\% sobre el total, con un crecimiento respecto de la vigencia anterior del 17.03\% consecuencia de la activación del embalse dando inicio a su depreciación a partir del mes de mayo de 2017, y para el caso del 2018 afecta todo el ejercicio contable". (amb, 2018, p.21)

\section{Indicadores de rentabilidad}

La utilidad neta sobre los ingresos totales "registra para la presente vigencia un valor de 18,93\%, se observa un incremento porcentual frente al año anterior del 3,25\% que se debe específicamente por la disminución en la provisión del impuesto de renta y sobretasa y el control de costos y gastos a lo largo de la vigencia” (amb, 2018, p.28) 


\section{Análisis del comportamiento económico de la empresa}

Es importante resaltar que la organización está realizando una buena utilización de sus recursos, provenientes en más de un $92 \%$ de las tarifas cobradas por consumo y cargo fijo de sus usuarios, las cuales se encuentran dentro de la normativa legal vigente, impuesta cada año por la resolución del CRA. En la empresa se evidencia un crecimiento en la utilidad neta obtenida al final de cada periodo contable desde 2014 y un patrimonio en crecimiento en los últimos tres años analizados. Todo lo anterior demuestra un potencial de crecimiento para la institución y la capacidad para adquirir nuevas unidades estratégicas de negocios desde su sólido desarrollo financiero y su planeación estratégica. (amb, 2018)

Adicional a esto la organización ha ampliado el número de usuarios atendidos, con una alta tasa de satisfacción al cliente y un alto nivel de productividad, con 462 empleados, muy por encima de las demás empresas estudiadas (amb, 2018). Este factor permite el establecimiento de una posibilidad de crecimiento y ampliación a empresa triple A.

3.3 POTENCIAL DE DESARROLLO DE LA EMPRESA DE SERVICIO PÚBLICO TRIPLE A DEL ÁREA METROPOLITANA DE BUCARAMANGA, FRENTE AL DESARROLLO TERRITORIAL Y LA PROYECCIÓN A DISTRITO METROPOLITANO.

Tabla 1: Matriz DOFA

Matriz DOFA: Planificación
estratégica de la consolidación
de una empresa triple A en la
concepción a futuro de un
distrito metropolitano.

distrito metropolitano

\section{Fortalezas $(\mathrm{F})$}

Una empresa existente con la experiencia en la oferta con calidad de los tres servicios. (Piedecuestana de servicios)

Empresa cuyo socio mayoritario es la administración de la alcaldía del municipio (Piedecuestana de servicios)

Las empresas Amb y Piedecuestana de servicios, cuentan con un buen manejo financiero.

Una empresa con la visión de ampliar el portafolio de servicios prestados a partir de la adquisición de nuevas unidades estratégicas.

Una empresa con capital para invertir en ampliación de infraestructura (Amb).

La empresa Empas, cuenta con una gran experticia en la administración, transporte y tratamiento de aguas residuales.

La empresa Empas, Posee amplios conocimientos en el manejo del saneamiento básico, para el diseño y mantenimiento de las redes, así

\section{Debilidades (D)}

Falta de una buena gestión en el manejo de los residuos sólidos, principalmente en cuanto a su disposición final (El carrasco).

Falta de conciencia social y políticas claras, en cuanto a los procesos de reciclaje y manejo de las basuras a nivel doméstico e industrial.

No existe una empresa de aseo con la capacidad de ampliación a más de un municipio.

Empresas de servicios de aseo con poca solidez financiera (Emaf) 


\begin{tabular}{|c|c|c|}
\hline & $\begin{array}{l}\text { como también el mapa de } \\
\text { georreferencia de las redes de } \\
\text { alcantarillado. } \\
\text { La empresa Emab, presta un buen } \\
\text { servicio de aseo }\end{array}$ & \\
\hline $\begin{array}{l}\text { Oportunidades (O) } \\
\text { Reforma a los estatutos, } \\
\text { posibilitando a la Amb, la } \\
\text { prestación del servicio de } \\
\text { alcantarillado a Bucaramanga, } \\
\text { Floridablanca y girón, } \\
\text { pagando los derechos de } \\
\text { interconexión a Empas. } \\
\text { La empresa Amb, se proyecta } \\
\text { a futuro como triple A y triple } \\
\text { E, en el largo plazo. } \\
\text { Tecnificación del manejo de } \\
\text { residuos sólidos. } \\
\text { Crecimiento del área } \\
\text { metropolitana, hacia el sur } \\
\text { donde está la triple A } \\
\text { Piedecuestana de Servicios. }\end{array}$ & $\begin{array}{l}\text { Estrategias FO } \\
\text { Establecimiento de alianzas } \\
\text { estratégicas entre el Amb y la } \\
\text { empresa Empas de forma que le } \\
\text { permita al Amb, prestar los } \\
\text { servicios de acueducto y } \\
\text { alcantarillado. } \\
\text { Establecimiento de alianza } \\
\text { estratégica con la empresa Emab, } \\
\text { para que en convenio con esta se } \\
\text { pueda tecnificar el manejo de } \\
\text { residuos sólidos, a partir de la } \\
\text { inyección de capital por parte de la } \\
\text { Amb, ampliando así la capacidad de } \\
\text { la empresa y por ende su cobertura. } \\
\text { Creación empresa del estado, con } \\
\text { patrimonio y rentas separadas del } \\
\text { Área o distrito o municipios. }\end{array}$ & $\begin{array}{l}\text { Estrategias DO } \\
\text { Desarrollo de políticas claras en el manejo } \\
\text { de los residuos sólidos, minimizando el } \\
\text { impacto ambiental. } \\
\text { Desarrollo de políticas y programas de } \\
\text { reciclaje y manejo de residuos sólidos } \\
\text { efectivos. } \\
\text { Establecimiento de campañas de } \\
\text { conciencia social para el reciclaje y la } \\
\text { reutilización, de forma que se minimice la } \\
\text { cantidad de residuos que llegan a los } \\
\text { rellenos sanitarios. }\end{array}$ \\
\hline $\begin{array}{l}\text { Amenazas (A) } \\
\text { Cierre de pequeñas empresas, } \\
\text { que podrían ser absorbidas por } \\
\text { el Amb o por Piedecuestana de } \\
\text { Servicios. } \\
\text { Rechazo de las propuestas por } \\
\text { parte de la comunidad por la no } \\
\text { socialización de estas. } \\
\text { Rechazo de propuestas por } \\
\text { parte entes } \\
\text { gubernamentales. } \\
\text { Existencia de sindicatos }\end{array}$ & $\begin{array}{l}\text { Estrategias FA } \\
\text { Desarrollo de políticas, enfocadas a } \\
\text { la continuidad de los cargos y el } \\
\text { personal que labora en ellos, como } \\
\text { parte de un proceso de } \\
\text { aprovechamiento de las } \\
\text { competencias existentes. } \\
\text { Inclusión de la comunidad y } \\
\text { sindicatos en el planteamiento y } \\
\text { desarrollo de las propuestas y } \\
\text { planes de acción a desarrollar como } \\
\text { distrito metropolitano. }\end{array}$ & $\begin{array}{l}\text { Estrategias DA } \\
\text { Involucramiento de los entes } \\
\text { gubernamentales en el buen manejo de } \\
\text { residuos sólidos. } \\
\text { Desarrollo de proyectos de utilización de } \\
\text { subproductos industriales y propuestas de } \\
\text { incentivos. }\end{array}$ \\
\hline
\end{tabular}

Para el presente estudio se realiza una reunión con un grupo de expertos, los cuales establecen el escenario inicial para la investigación prospectiva, después de realizar el análisis de las empresas prestadoras de servicios públicos domiciliarios de acueducto, aseo y alcantarillado, más relevantes del sector.

Supuestos claves:

En el área metropolitana de Bucaramanga existe una proyección hacia la consolidación de un distrito metropolitano, que establece una posibilidad de Fusión o Unión temporal en sus instituciones prestadoras de servicios públicos domiciliarios.

En el AMB existe una empresa triple A (Piedecuestana de Servicios Públicos) y dos empresas que tienen proyección triple A (Acueducto Metropolitano de Bucaramanga -Amb y Empresa Pública de Alcantarillado de Santander EMPAS S.A E.S. P). 
La operación de las redes de alcantarillado de Bucaramanga, Floridablanca y Girón las tiene EMPAS en comodato, debido a que estas pertenecen al municipio y el Amb suministra agua a estos mismos municipios.

Para el presente estudio los expertos determinan que la empresa con una mayor proyección a futuro a empresa triple A, es el Acueducto Metropolitano de Bucaramanga -Amb, debido a que este posee una importante infraestructura que le permite una amplia cobertura del servicio, la cual ha presentado un crecimiento importante en los últimos años, sin disminuir su capacidad empresarial, garantizando un suministro de agua constante en los sectores atendidos.

El Amb cuenta también con una dirección estratégica adecuada que ha llevado a la empresa a alcanzar niveles deseables de rentabilidad. Es importante establecer que el aspecto más relevante que presenta para proyectar el Amb a triple A, es la declaración desde su planeación estratégica de crecimiento y adquisición de nuevas unidades estratégicas de negocios y el establecimiento de alianzas para el logro de estos objetivos.

\section{Potencial de desarrollo de la empresa de acueducto metropolitano de Bucaramanga Amb}

Para establecer el potencial de desarrollo de la empresa de acueducto metropolitano de Bucaramanga Amb, se realizó un análisis estructural, el cual es una herramienta diseñada para el enlace de ideas. Este Permitió describir el sistema gracias a una matriz que integra a todos sus elementos constitutivos. El método se habilitó, estudiando estas relaciones, encontrando las variables influyentes, dependientes y esenciales para entender la evolución del sistema y predecir su comportamiento futuro. (Ballesteros Rivero \& Ballestero Silva, 2008)

Para el presente análisis se utilizó "El método estructural MICMAC, el cual es un instrumento diseñado para vincular ideas y se apoya en el juicio cualitativo de actores y/o expertos en determinado asunto, el cual permite describir el sistema desde una matriz que une todos sus componentes. (Gómez Benjumea, 2016, p.6).

El presente estudio está dividido en tres fases:

Fase 1: listado de las variables del sistema

Fase 2: la descripción de relaciones entre variables del sistema.

Fase 3: la identificación de variables clave y sus categorías

Tabla 2. Definición del grupo de expertos

\begin{tabular}{|c|c|}
\hline Área de estudio & Cargo \\
\hline Magister en Administración-MBA & Docente Investigador- UNC \\
\hline Especialista en Gerencia de Proyectos de Construcción & Subgerente de Tratamiento Integral - EMPAS \\
\hline
\end{tabular}




\begin{tabular}{|c|c|}
\hline Magister en Administración Publica & Servicio al Cliente - EMPAS \\
\hline Economía & Docente ESAP \\
\hline Especialista en Gerencia de Calidad & Profesional 2 de Servicio al Cliente - EMPAS \\
\hline Especialista Derecho Administrativo & $\begin{array}{c}\text { Profesional Universitaria de Atención al Usuario - } \\
\text { Piedecuestana de Servicios }\end{array}$ \\
\hline
\end{tabular}

Definición Macro-Sistemas: El sector servicios públicos domiciliarios de Bucaramanga y amb, está regulado por: Superintendencia de Servicios Públicos Domiciliarios, Alcaldía de Bucaramanga, Alcaldía de Floridablanca., Alcaldía de Girón, Alcaldía de Piedecuesta, Dirección técnica de gestión de acueducto y alcantarillado, Dirección de Gestión de aseo.

Definición de los subsistemas: Los principales subsistemas son: políticas de servicios públicos domiciliarios, entidades departamentales, entidades municipales, proyección a distrito, infraestructura tecnología, equipo tecnológico, redes de trabajo, conocimiento-innovación, talento humano, recursos, usuarios.

Definición de las variables: Infraestructura, Direccionamiento estratégico de la organización, Satisfacción del cliente, Desarrollo del talento humano, Rentabilidad, Normatividad vigente, Cobertura del servicio, Capacidad empresarial, Sistema tarifario, Alianzas estratégicas, Proyección a distrito, Proyección a triple AAA 
Tabla 3. Descripción de variables

\begin{tabular}{|c|c|c|c|c|}
\hline $\mathbf{N}^{\circ}$ & Long label & Short label & Description & Theme \\
\hline & Infraestructura & Inf & $\begin{array}{l}\text { Conjunto de medios técnicos, servicios e instalaciones } \\
\text { necesarios para el desarrollo de la actividad de una organización }\end{array}$ & Técnico \\
\hline & $\begin{array}{l}\text { Direccionamiento } \\
\text { estratégico }\end{array}$ & DirE & $\begin{array}{l}\text { Es una disciplina ejercida por los directivos, que a partir de la } \\
\text { planeación define la orientación de una empresa a partir de las } \\
\text { oportunidades y amenazas del entorno y de los recursos con que } \\
\text { esta cuenta. }\end{array}$ & Administrativo \\
\hline & Satisfacción del cliente & S.C & $\begin{array}{l}\text { Grado en el que el usuario de un producto o servicio percibe que } \\
\text { se cumple con sus expectativas. }\end{array}$ & Administrativo \\
\hline & $\begin{array}{l}\text { Desarrollo del talento } \\
\text { humano }\end{array}$ & TH & $\begin{array}{l}\text { Proceso mediante el cual una organización garantiza la } \\
\text { formación en sus colaboradores de las habilidades, destrezas, } \\
\text { actitudes y conocimientos, de forma que le permitan el } \\
\text { desarrollo idóneo de las actividades requeridas en una } \\
\text { organización. }\end{array}$ & Administrativo \\
\hline & Rentabilidad & Rent & $\begin{array}{l}\text { Relación entre los beneficios que genera una organización y la } \\
\text { inversión realizada. }\end{array}$ & Administrativo \\
\hline & Normativa Vigente & Norm & $\begin{array}{l}\text { Normativa que rige una actividad empresarial y que forma parte } \\
\text { del macro entorno de la misma. }\end{array}$ & Legal \\
\hline & Cobertura del servicio & C.Serv & $\begin{array}{l}\text { Es la medida en que las empresas proporcionan los servicios en } \\
\text { las zonas que requieren los usuarios. Se establece como el } \\
\text { porcentaje de predios residenciales con acceso al servicio. }\end{array}$ & Técnico \\
\hline & Capacidad empresarial & Cap & $\begin{array}{l}\text { Se define como el conjunto de condiciones y aptitudes que } \\
\text { permiten el desarrollo de forma eficiente y eficaz de los servicios } \\
\text { prestados dentro de la cobertura empresarial incluyendo su } \\
\text { crecimiento a futuro. }\end{array}$ & Técnico \\
\hline & Sistema tarifario & S.Tar & $\begin{array}{l}\text { Se define como el precio pagado por los usuarios o } \\
\text { consumidores de un servicio público al estado o concesionario a } \\
\text { cambio de la prestación de un servicio. Esta se fija teniendo en } \\
\text { cuenta los costos de prestación del servicio y la normativa } \\
\text { vigente. }\end{array}$ & Administrativo \\
\hline & Alianzas estratégicas & A.estr & $\begin{array}{l}\text { Acuerdo realizado por dos o más partes, para alcanzar un } \\
\text { conjunto de objetivos deseados por cada parte } \\
\text { independientemente. Entre esta se encuentran las uniones } \\
\text { temporales, fusiones y adquisiciones. }\end{array}$ & Administrativo \\
\hline
\end{tabular}

Se realizó la respectiva valoración de la Motricidad o Influencia de las variables, sobre el sistema.

\section{Calificación de las relaciones entre las variables}

Niveles de fuerza de la variable. Se establecieron los rangos de influencias en el software MICMac para las variables que se definieron al principio del estudio, el cual va de 0 a 3 según el nivel de influencia que tenga una variable en la otra. 
Figura 1. Plano de influencias y dependencias directas Influences range from 0 to 3 , with the possibility to identify potential influences: 0 : No influence

1: Weak

2: Moderate influence

3: Strong influence

P: Potential influences

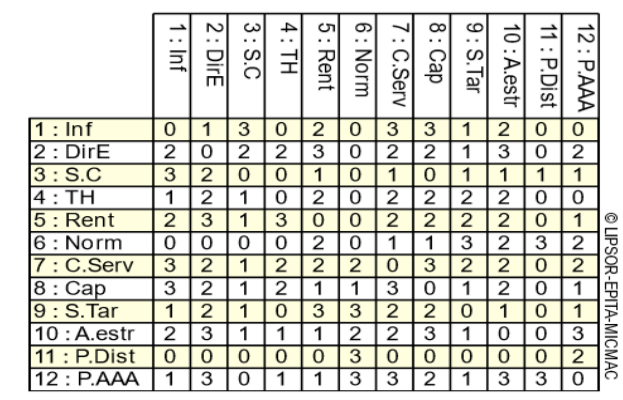

Fuente: MIC-MAC

En la figura se observa el Plano de influencias y dependencias directas, para el cual se establecieron cuatro rangos, donde 0 es ninguna influencia, 1 influencia débil, 2 influencia moderada y 3 influencia fuerte. Dicho plano se realizó contando con la opinión de expertos en el tema, y posteriormente se procedió a la elaboración del plano de influencias y dependencias directas.

Figura 2. Plano de influencias y dependencias directas

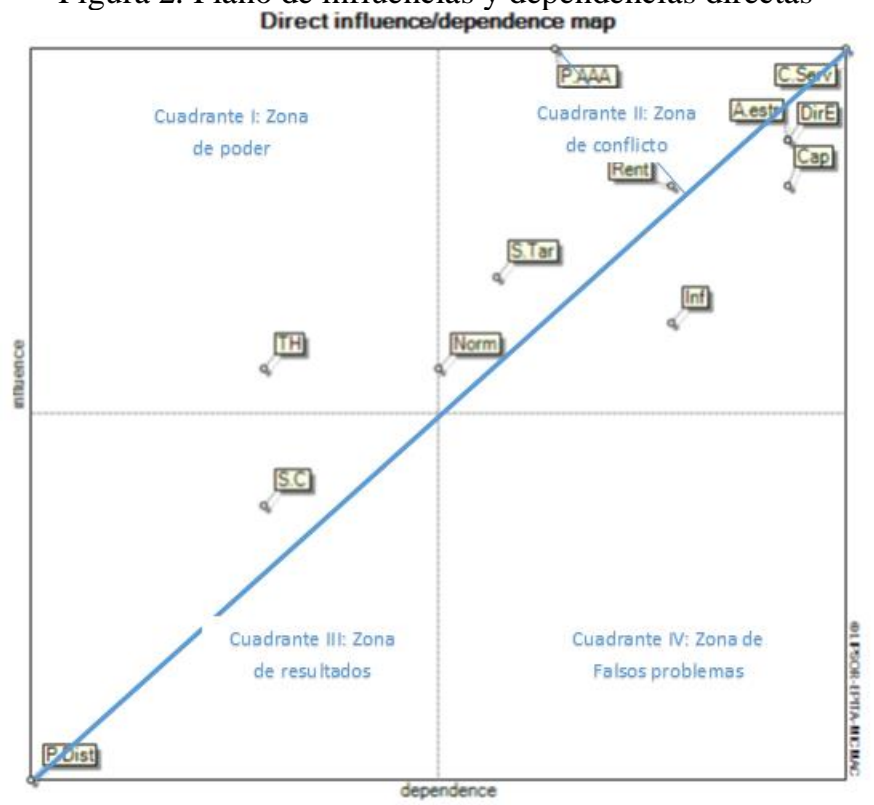

Fuente: MIC-MAC

Cuadrante I: Zona de poder. Las variables localizadas en este cuadrante son tipo AB, "altamente" motrices sobre el sistema, pero poco "dependientes" del sistema. Es decir, no están bajo el control del sistema. En este cuadrante se encuentra la variable desarrollo del talento humano, esta presenta una alta 
influencia y baja dependencia, esto implica que es autónoma y seguirá su funcionamiento, aun cuando la organización no se enfoque en esta.

Cuadrante II: Zona de conflicto. Las variables localizadas en este cuadrante son tipo AA, “altamente" motrices sobre el sistema, pero también altamente “dependientes” del sistema. Es decir, están bajo el control del sistema. En este cuadrante se encuentran ubicadas las siguientes variables cobertura de servicio, direccionamiento estratégico, alianzas estratégicas, capacidad empresarial, rentabilidad, proyección AAA, infraestructura, sistema tarifario y normatividad son variables de alta dependencia e influencia en la organización. Dichas variables son bastante volátiles y si la empresa no trabaja sobre ellas, están van a afectar la organización. Por esta razón la institución debe actuar sobre ellas de forma inmediata, porque están bajo el control del sistema. Por lo tanto, hay que desarrollar estrategias de muy corto plazo para afectarlas.

Cuadrante III: Zona de resultados. Las variables localizadas en este cuadrante son tipo BA, de "baja" motricidad sobre el sistema, pero altamente dependientes" del sistema. Es decir, están bajo el control del sistema, pero con poca motricidad, en este caso se encuentran las variables

Proyección a distrito y servicio al cliente, estas presentan baja influencia y poca dependencia, razón por la cual no son relevantes para la organización en su proyección a futuro

Variables estratégicas: Se determina que las variables estratégicas de la organización, las cuales presentan mayor volatilidad y son, Cobertura de servicio, direccionamiento estratégico, alianzas estratégicas, capacidad empresarial, proyección AAA rentabilidad.

Figura 3. Plano de desplazamientos directo/indirecto Displacement map : directindirect

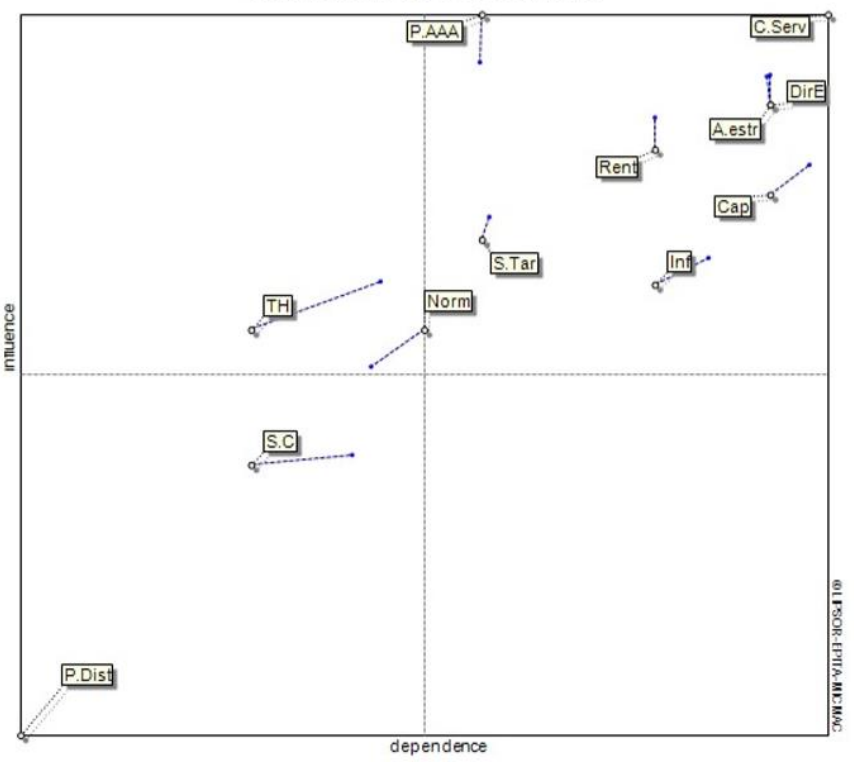

Fuente: MIC-MAC 
En la figura se muestra el plano de desplazamientos de las variables a estudio, donde se observa que estas en general presentan una baja movilidad, son embargo se observa que la normatividad vigente se desplazaría del cuadrante I zona de conflicto al cuadrante II zona de poder.

Construcción de escenarios:

Tabla 4. Supuestos para los escenarios:

\begin{tabular}{|c|c|c|c|c|}
\hline Variable & Supuesto I & Supuesto II & Supuesto III & Supuesto VI \\
\hline $\begin{array}{l}\text { Cobertura } \\
\text { de servicio }\end{array}$ & $\begin{array}{c}\text { Decrecimiento } \\
\text { de la cobertura } \\
\text { de servicio. }\end{array}$ & $\begin{array}{l}\text { Debilitamiento en el } \\
\text { crecimiento de la } \\
\text { cobertura del } \\
\text { servicio }\end{array}$ & $\begin{array}{c}\text { Aumento } \\
\text { significativo en la } \\
\text { cobertura del } \\
\text { servicio. }\end{array}$ & $\begin{array}{c}\text { Nivel de } \\
\text { cobertura del } \\
100 \%\end{array}$ \\
\hline $\begin{array}{l}\text { Direcciona } \\
\text { miento } \\
\text { estratégico }\end{array}$ & $\begin{array}{c}\text { El } \\
\text { direccionamient } \\
\text { o estratégico es } \\
\text { desfavorable } \\
\text { para la } \\
\text { organización y } \\
\text { la conduce a un } \\
\text { retroceso en su } \\
\text { visión. }\end{array}$ & $\begin{array}{l}\text { El direccionamiento } \\
\text { estratégico de la } \\
\text { organización } \\
\text { mantiene la } \\
\text { organización en el } \\
\text { tiempo en sus } \\
\text { condiciones } \\
\text { iniciales. }\end{array}$ & $\begin{array}{l}\text { La organización } \\
\text { cuenta con un } \\
\text { excelente } \\
\text { direccionamiento } \\
\text { estratégico. }\end{array}$ & $\begin{array}{c}\text { El } \\
\text { direccionami } \\
\text { ento } \\
\text { estratégico ha } \\
\text { posicionado } \\
\text { la empresa } \\
\text { como la } \\
\text { mejor a nivel } \\
\text { nacional }\end{array}$ \\
\hline $\begin{array}{l}\text { Alianzas } \\
\text { estratégicas }\end{array}$ & $\begin{array}{c}\text { La empresa no } \\
\text { tiene la } \\
\text { intención de } \\
\text { asumir alianzas } \\
\text { estratégicas }\end{array}$ & $\begin{array}{l}\text { La empresa asumirá } \\
\text { alianzas estratégicas } \\
\text { en el largo plazo }\end{array}$ & $\begin{array}{c}\text { La empresa } \\
\text { asumirá alianzas } \\
\text { estratégicas en el } \\
\text { corto plazo }\end{array}$ & $\begin{array}{c}\text { La } \\
\text { organización } \\
\text { asume } \\
\text { alianzas } \\
\text { estratégicas } \\
\text { para su } \\
\text { beneficio }\end{array}$ \\
\hline
\end{tabular}

Escenarios: Para la construcción de escenarios el estudio se basa en la información tomada de la organización y la opinión de los expertos, adicional a esto, los supuestos se construyen a partir de la información encontrada en la revisión de literatura e informes de las organizaciones.

Supuesto I: Hace referencia a un escenario pesimista, este se consolida como un esta que es completamente indeseable para el Amb, en el cual confluyen situaciones adversas de orden interno y externo. Nombre de escenario "obscuridad total".

Supuesto II: Hace referencia al escenario tendencial, este es aquel en el que el Amb no realiza ningún tipo de esfuerzo, por alcanzar el mejoramiento de las condiciones iniciales. Nombre de escenario "Staus Quo".

Supuesto III: Se define como un escenario cercano al ideal, este es bastante posible de lograr si se centran los esfuerzos en el crecimiento empresarial de forma rentable, este escenario es nombrado "Escenario Platino".

Supuesto IV: Es el escenario más optimista para el Amb, es un estado ideal pero aun asa difícil de alcanzar, para tal fin sea nombrado como Escenario triple A (Apuesta). 
Escenario obscuridad total:

Para 2025, el Amb asume un direccionamiento estratégico desfavorable para la organización, lo que la conduce a un retroceso en su visión, por lo que incurre en un decrecimiento de su rentabilidad. Adicional a esto la empresa se niega a adquirir alianzas estratégicas que podrían ayudarla a solventar sus problemas, de forma que se mantiene un decrecimiento de la cobertura de servicio y la capacidad empresarial, haciendo imposible la adquisición de nuevas unidades estratégicas de negocios y por ende su proyección a triple A.

\section{Escenario Staus Quo:}

El direccionamiento estratégico de la organización mantiene el Amb para 2025 en sus condiciones iniciales en cuanto a su rentabilidad, manteniendo constátate la capacidad empresarial y un crecimiento ralentizado en la cobertura del servicio. La organización establece en su planeación estratégica la proyección a triple A, por lo que plantea el desarrollo de alianzas estratégicas en el largo plazo.

\section{Escenario Platino:}

En el 2025, el Acueducto metropolitano de Bucaramanga S.A. E.S.P cuenta con un excelente direccionamiento estratégico, que ha llevado a la organización a un aumento significativo en la cobertura del servicio y un incremento de la capacidad empresarial y la rentabilidad, por tal motivo la institución estableció la necesidad de establecer alianzas estratégicas a corto plazo con el fin de adelantar esfuerzos encaminados a la proyección triple A.

\section{Escenario triple A (Apuesta):}

El excelente direccionamiento estratégico que ostenta el Amb ha llevado a la organización a posicionarse como la mejor a nivel nacional en el año 2025, a partir de la adquisición de nuevas unidades estratégicas de negocio, que le permitieron el establecimiento como empresa triple A, con la ayuda de alianzas institucionales, (Empas y empresas de aseo), lo que le ha permitido alcanzar una cobertura del $100 \%$ en los municipios de Bucaramanga, Florida y Girón y un suministro continuo de sus servicios las 24 horas, los 365 días del año. Adicional a esto la empresa incremento rentabilidad a partir del aumento de su nivel de Utilidad Operacional sobre el promedio de los últimos años, y ha fortalecido sus activos, y se evidencia un mejoramiento de Utilidad Neta sobre los Ingresos Operacionales. 


\section{CONCLUSIONES}

Desde la caracterización de las empresas prestadoras de servicios públicos en el área Metropolitana de Bucaramanga, se observa que existe una empresa donde su estructura organizacional cumple con ser triple A y su estructuración de personal es clara, equitativa y sus manejos financieros son estables y la cual desde su cumplimiento como empresa de carácter público, única en el nororiente colombiano, presenta información para el público y ciudadanía y responde a los requerimientos de transparencia, la Piedecuestana de Servicios Públicos, empresa única con experiencia en el manejo administrativo, logístico y de distribución de servicios de Aseo, acueducto y alcantarillado, para un municipio como Piedecuesta que está en crecimiento poblacional y donde ha sido capaz de responder eficientemente con su oferta.

En el caso de Bucaramanga se consolida una gran empresa con proyección triple A, como es la empresa Acueducto metropolitano de Bucaramanga, que presta los servicios de acueducto con una gran cobertura y capacidad en Bucaramanga, Floridablanca y Girón. En cuanto al caso del servicio de alcantarillado, este es prestado en comodato por la empresa EMPAS, en el área metropolitana y a lo largo del departamento de Santander, es importante resaltar que esta empresa es clave para las alianzas estratégicas que debe desarrollar el amb en su consolidación a empresa triple A, es decir esta empresa busca establecer alianzas estratégicas para ofrecer los servicios de alcantarillado y aseo en toda el Área Metropolitana de Bucaramanga, por esto fue objeto de estudio de prospectiva.

Las demás empresas del AMB tienen especialidad en una sola prestación de servicio como EMPAS o son administradoras de las transferencias (EMAF). Otros municipios organizan la prestación de sus servicios en pequeñas empresas que por su tamaño no fueron objeto de este estudio, las cuales no poseen la capacidad para la prestación de un buen servicio como es el caso de Girón que cuenta con cinco empresas de aseo, entre las que se encuentran, Aseo Ruitoque, Proactiva Chicamocha y Caralimpia, Econatural y limpieza Urbana que no consiguen realizar procesos coordinados para establecer buenas rutas de recolección y barrido, creando la necesidad de contar con una empresa municipal.

La prestación de servicio de aseo en Bucaramanga cuenta con cuatro empresas: REDIBA, Limpieza Urbana, Proactiva Chicamocha y EMAB, donde esta última posee el $82 \%$ de la cobertura en Bucaramanga. Pese a los múltiples problemas y demandas por la utilización del carrasco y por qué en el municipio de Bucaramanga actualmente solo se realiza un reciclaje formal del dos por ciento, se observa que esta empresa tiene deseo de solucionar sus inconvenientes y aumentar su capacidad, por ello adquirió una moderna Estación de Clasificación y Aprovechamiento de Residuos Sólidos, consolidándose como una buena aliada estratégica para la prestación de los servicios triple A en el área metropolitana. 
En cuanto al componente técnico la empresa Piedecuestana de Servicios responde como una empresa triple A, con gran organización en la prestación de estos servicios, que opera de forma rentable, con buena cobertura, capacidad y servicio al cliente; su carácter de empresa pública le permite a la administración municipal injerir y asumir direccionamiento en momentos de crisis. Esta empresa no requirió hacerle prospectiva a triple A porque en la actualidad lo es, evidenciando su efectividad en la oferta de sus servicios frente a la demanda desde lo técnico, financiero y talento humano.

Después de realizar el análisis del comportamiento económico de la empresa de servicio público con proyección triple A del Área Metropolitana de Bucaramanga, frente al desarrollo territorial y la proyección a distrito metropolitano, se establece que la empresa del Acueducto metropolitano de Bucaramanga tiene la capacidad y solidez financiera para la realización de alianzas estratégicas (Principalmente con la empresa EMPAS), que permitirían en un futuro consolidar esta empresa como triple A en los municipios de Bucaramanga, Girón y Floridablanca, para una posterior fusión o unión temporal con la Piedecuestana de servicios públicos, quien tiene la experiencia del manejo de los tres servicios en el municipio de Piedecuesta.

Desde el análisis prospectivo, se evidencia que el potencial de desarrollo de la empresa de Acueducto Metropolitano de Bucaramanga tiene una proyección triple A frente al desarrollo territorial y a distrito metropolitano, se demuestra la necesidad de centralización en la prestación de los servicios de forma que se permita la reducción de los costos de operación y gastos administrativos, así como también el aumento de la eficacia a partir de una única planeación estratégica. Al realizar la construcción de escenario se determinó que la empresa lograra los mejores resultados a futuro a partir del establecimiento de buenas alianzas estratégicas, es el mejor camino para las proyecciones que establece desde su propósito empresarial para la expansión a prestar servicios de aseo y alcantarillado, y se contaría con una sola entidad de gobierno central con un único concejo y dirección, reduciendo los costos administrativos.

El proceso de avance a distrito metropolitano y la proyección de empresas de servicios públicos triple A, implica un proceso de participación ciudadana en los municipios del AMB, donde se debe reconocer que Bucaramanga cuenta con el 50\% de población en lo urbano pero el otro 50\% está en los municipios aledaños y que cuentan con proyección de crecimiento poblacional, luego el reconocimiento de ventajas frente a distrito o consolidación de empresas triple A, debe contarse con apoyo ciudadano, además que estas comunidades como el caso de Piedecuesta saben que cuentan cuenta con fuentes hídricas para abastecer acueducto y han defendido con cabildos su manejo; los demás municipios dependen de fuentes que provienen de otros municipios como los de la provincia de soto norte o Lebrija y por ello han liderado procesos en contra de la minería a gran escala. 
En cuanto al componente político para avanzar hacia la consolidación de distrito metropolitano, se requiere de un apoyo de la comunidad y donde los dirigentes desarrollen estrategias de generación de confianza en la región y se busquen desarrollos visionarios que proyecten la región no solo nacional, sino internacionalmente y se definan áreas de trabajo con responsabilidad social y transparencia en sus manejos. Todo lo anterior a partir de estudios de diagnóstico y el establecimiento de objetivos y sus estrategias y planes de acción para su desarrollo.

Entre las posibilidades para consolidar el distrito metropolitano, se hace necesario consolidar el área metropolitana con proyectos de beneficio e integración territorial, que generen confianza en la comunidad y donde la transparencia y servicio al cliente, atendiendo situaciones críticas para la comunidad, generando responsabilidad social desde lo económico, ambiental y social, como motor de impulso regional desde las empresas prestadoras de servicios públicos.

Las empresas prestadoras de servicios públicos de acueducto, aseo y alcantarillado deben desde su planeación estratégica, con estudios prospectivos, visionar los diferentes escenarios para buscar su adaptación a los cambios que se generarían a partir de la posibilidad de consolidación de distrito metropolitano.

Es importante que las empresas acueducto metropolitano de Bucaramanga, EMPAS S.A. E.S.P, EMAB, consideren en establecer una unión temporal o fusión, de forma que les permita el desarrollo organizacional desde una mayor capacidad para afrontar los desafíos que conllevan la consolidación de un distrito metropolitano, o pensar en una alianza estratégica en proyección de una empresa Triple A y para ello se debe contar con la experticia y manejo de la Piedecuestana de Servicios públicos.

Adicional a esto se debe apoyar la consolidación de un distrito metropolitano, debido a que dicho propósito permitirá un mayor desarrollo de la región, así como también mayor equidad y la posibilidad de desarrollar una mayor cantidad de proyectos que apunten a mejorar la calidad de vida de las personas a partir de la asignación de mejores presupuestos, diminución en los gastos administrativos y la unificación en la planeación y gestión de los recursos.

Frente al gran desafío de un distrito metropolitano, es fundamental revisar como el área metropolitana realiza procesos de generación de cultura ciudadana en el manejo de residuos sólidos desde la fuente y donde esta cultura se refleje en las empresas prestadoras de estos servicios con la generación de empresas transformadoras de los mismos, donde además no solo se generen empresas, sino puestos de trabajo que involucren las diferentes comunidades del área. 


\section{REFERENCIAS}

Alcaldia de Bucaramanga. (30 de octubre de 2019). En proceso de empalme se ratificó compromiso con el aprovechamiento de los residuos en Bucaramanga. Recuperado el 5 de noviembre de 2019, de Redes sociales: https://www.bucaramanga.gov.co/noticias/en-proceso-de-empalme-se-ratifico-compromisocon-el-aprovechamiento-de-los-residuos-en-bucaramanga/

Alcaldía municipal de Floridablanca. (2014). Acta de informe de gestion. Recuperado el 20 de octubre de 2019, http://www.floridablanca.gov.co/Transparencia/ControlyRendiciondeCuentas/Acta\%20de\%20Informe\% 20de\%20Gesti\%C3\%B3n\%20\%20GABRIEL\%20TORRA.pdf

Amb. (2014). Carta a los Accionistas. Recuperado el 30 de octubre de 2019, de http://www.amb.com.co/frmInformacion.aspx?inf=129

Amb. (2018). Informe de gestion de la empresa Acueducto Metropolitano de Bucaramanga. Recuperado $\begin{array}{llllll}\text { el } 24 & \text { de } & \text { septiembre }\end{array}$ http://www.amb.com.co/DocumentoInfo/INFORME_GESTION_2018.pdf

Ballesteros Rivero, D., \& Ballestero Silva, P. (septiembre de 2008). Análisis Estructural Prospectivo Aplicado al Sistema Logístico. Scientia Et Technica, 2(39). Recuperado el 5 de Octubre de 2019, de $\begin{array}{llll}\text { Scientia et } & \text { Technica Xño XIV: }\end{array}$ https://revistas.utp.edu.co/index.php/revistaciencia/article/view/3197/1829

Banco Interamericano de Desarrollo. (2015). Las ciudades intermedias con mayor potencial en Colombia. Recuperado el 16 de octuvre de 2019, de file:///C:/Users/USUARIO/Downloads/Las-ciudadesintermedias-con-mayor-potencial-en-Colombia-Un-sistema-de-identificaci\%C3\%B3n.pdf

Blu Radio Bucaramanga. (8 de noviembre de 2018). Multan a la EMAB por fallas en la disposición final de basuras en El Carrasco. Recuperado el 3 de noviembre de 2019, de https://www.bluradio.com/bucaramanga/multan-la-emab-por-fallas-en-la-disposicion-final-de-basurasen-el-carrasco-196186-ie3509886

Carrión Barrero, G. A. (2009). Los avatares de la institucionalidad metropolitana en Colombia: una breve revisión a la aplicación de la Ley 128 de 1994. Recuperado el 16 de octubre de 2019, de file:///C:/Users/USUARIO/Downloads/435-1522-1-PB.pdf

Cely, A. (1999). Metodología de los Escenarios para Estudios. Recuperado el 10 de octubre de 2019, de Revista Ingeniería e Investigación No. 44: http://www.bdigital.unal.edu.co/24172/1/21296-72403-1PB.pdf

Comisión Regional de Competitividad Santander Competitivo. (2013). Hacia un modelo sostenible de gobernabilidad en el AMB. Recuperado el 18 de deptiembre de 2019, de https://www.camaradirecta.com/media/bb9ddca06d2e9bed2a632d37c719f3fed889a1e1.pdf 
EMPAS. (2019). Pagina web de la empresa Empas. Recuperado el 10 de octunre de 2019, de https://www.empas.gov.co/

ESANT. (1 de Noviembre de 2018). Análisis interno de la empresa (Perfil de capacidad institucional). $\begin{array}{llllll}\text { Recuperado el } & 23 & \text { de } & \text { Septiembre } & \text { de }\end{array}$ file:///C:/Users/USUARIO/Downloads/ANALISIS\%20INTERNO\%20ESANT\%20SA\%20ESP.pdf

European Commiission. (Mayo de 2010). Mapping Of The Public Services. Recuperado el 10 de octubre de 2019, de Public Services in The European Union \& in the 27 members states: https://www.ceep.eu/images/stories/pdf/Mapping/CEEP_mapping\%20experts\%20report.pdf

Gómez Benjumea, L. A. (2016). Metodología prospectiva "Herramientas de prospectiva". Obtenido de https://www.academia.edu/27659450/EJERCICIO_DE_PROSPECTIVA_CON_MIC_MAC?auto=dow nload

Piedecuestana de servicios públicos e.s.p. (2019). Pagina web Piedecuestana de servicios públicos e.s.p. Recuperado el 20 de septiembre de 2019, de https://www.piedecuestanaesp.gov.co/

Ramírez J, J. C., \& de Aguas P, J. m. (Diciembre de 2015). Cepal. Recuperado el 12 de septiembre de 2018, de Escalafón de la competitividad de los departamentos de Colombia 2015: https://repositorio.cepal.org/bitstream/handle/11362/39627/1/S1501237_es.pdf

Suárez, P. M. (07 de 2021). Ciudad Creativa. Ciencias sociales para ciudadanos del Siglo XXI. Proyecto educativo. South Florida Journal of Development, 25. doi:10.46932/sfjdv2n3-038

Universidad Industrial de Santander. (2018). Plan integral de residuos sólidos de Area Metrpolitana de Bucaramanga. Recuperado el 01 de octubre de 2020, de chromeextension://ohfgljdgelakfkefopgklcohadegdpjf/http://versionantigua.bucaramanga.gov.co/documents/de pendencias/DIAGNOSTICO\%20FINAL\%20COMPILADO.pdf 